\title{
Are the Variants of the Circle of Willis Determined by Genetic or Environmental Factors? Results of a Twin Study and Review of the Literature
}

\author{
Bianka Forgo, ${ }^{1}$ Adam Domonkos Tarnoki, ${ }^{1,2}$ David Laszlo Tarnoki, ${ }^{1,2}$ Daniel Tamas Kovacs, ${ }^{1}$ \\ Laszlo Szalontai, ${ }^{1}$ Aliz Persely, ${ }^{1}$ Anita Hernyes, ${ }^{1}$ Marcell Szily, ${ }^{1}$ Levente Littvay, ${ }^{3}$ Emanuela Medda, ${ }^{4}$ \\ Adam Szabo, ${ }^{5}$ Lajos Rudolf Kozak, ${ }^{5}$ Gabor Rudas, ${ }^{5}$ Attila Sas, ${ }^{6}$ Marianna Sepsi, ${ }^{7}$ Laszlo Kostyal, $^{7}$ \\ and Csaba Olah ${ }^{8,9}$ \\ ${ }^{1}$ Department of Radiology, Semmelweis University, Budapest, Hungary \\ ${ }^{2}$ Hungarian Twin Registry, Budapest, Hungary \\ ${ }^{3}$ Central European University, Budapest, Hungary \\ ${ }^{4}$ Centre for Behavioural Sciences and Mental Health, Istituto Superiore di Sanità, Rome, Italy \\ ${ }^{5}$ MR Research Centre, Semmelweis University, Budapest, Hungary \\ ${ }^{6}$ Department of Neurology, Borsod County University Teaching Hospital, Miskolc, Hungary \\ ${ }^{7}$ Department of Radiology, Borsod County University Teaching Hospital, Miskolc, Hungary \\ ${ }^{8}$ Department of Neurosurgery, Borsod County University Teaching Hospital, Miskolc, Hungary \\ ${ }^{9}$ Department of Neurology, University of Debrecen, Debrecen, Hungary
}

\begin{abstract}
Background: Anatomic variants of the circle of Willis (CW) are commonly observed in healthy subjects. Genetic and environmental factors influencing these variants remain unclear. Our aim was to assess the genetic and environmental background affecting variant CW phenotypes. Methods: A total of 122 adult healthy twins from the Hungarian Twin Registry (39 monozygotic (MZ) and 22 dizygotic (DZ) pairs, average age $49.7 \pm 13.4$ years) underwent Time-of-Flight magnetic resonance angiography and transcranial Doppler sonography. We investigated the anterior and posterior CW according to morphological categories. Prevalence and concordance rates of CW variants were calculated. MZ twins discordant for $\mathrm{CW}$ variants were analyzed for cardiovascular risk factors and altered blood flow. Results: Complete CW (45.0\%) and bilaterally absent posterior communicating artery (PCoA) (22.5\%) were the most prevalent variants in the anterior and posterior $\mathrm{CW}$, respectively. There was no significant difference regarding the prevalence of variants across zygosity except for bilaterally hypoplastic PCoA $(p=.02)$. DZ concordance was higher compared to $\mathrm{MZ}$ twins regarding morphological categories of the $\mathrm{CW}$. Cardiovascular risk factors were not significantly associated with variant $\mathrm{CW}$ in $\mathrm{MZ}$ twins discordant to $\mathrm{CW}$ morphology. Flow parameters did not differ significantly among $\mathrm{MZ}$ twins discordant to $\mathrm{CW}$ variants. Conclusion: $\mathrm{CW}$ variants may not be determined by substantial genetic effects and are not influenced by altered blood flow in healthy individuals. Further investigations are needed to identify potential environmental factors affecting these variants.
\end{abstract}

Keywords: Circle of Willis, variant, genetic, twin, cerebrovascular variants, collateral flow, cerebrovascular development

The circle of Willis (CW), an arterial anastomotic polygon located at the skull base, is of key importance regarding the maintenance of cerebral perfusion. Variations of the CW are common findings in healthy individuals and are associated with a greater risk of stroke in case of hypoplastic or absent segments of the CW. Prospective studies have confirmed that incomplete anterior CW is significantly associated with anterior circulation stroke in patients without prior cerebrovascular disease (van Seeters et al., 2015), and
ReCEIVED 26 April 2018; ACCEPTED 2 August 2018. First published online 11 September 2018.

ADDRESS FOR CORRESPONDENCE: Bianka Forgo, Department of Radiology, Semmelweis University, 78/a Üllői street, Budapest H-1082, Hungary. E-mail: fbia021@gmail.com

This paper was presented at the Twins 2017 Congress, Madrid, Spain, on November 17, 2017, which is the Joint 4th World Congress on Twin Pregnancy and 16th Congress of the International Society of Twin Studies (ISTS). 
the risk is even higher if incomplete posterior CW is present simultaneously (van Seeters et al., 2015). Non-functional anterior and posterior collaterals have a higher prevalence in patients with anterior circulation stroke compared to patients with asymptomatic carotid artery disease (Hoksbergen, Legemate et al., 2003). The collateral flow in the $\mathrm{CW}$ has been widely investigated in patients with internal carotid artery (ICA) occlusion or stenosis, and intact collaterals seem to have a key role in maintaining cerebral perfusion in this patient group (Bisschops et al., 2003; Henderson et al., 2000; Hendrikse et al., 2001; Kluytmans et al., 1999; Qiu et al., 2015; Rutgers et al., 2004; Schomer et al., 1994) as the collaterals may compensate for the restricted flow in the stenotic vessel, which is the major artery ensuring cerebral blood supply (Morgenstern et al., 1997; Tegos et al., 2000). Other neurological conditions have been investigated in relation to $\mathrm{CW}$ variants as well, emphasizing the significance of CW variants. Aneurysms and their recurrence after endovascular coiling are more common with variant CW (Songsaeng et al., 2010). There might be an association between the variant CW anatomy and white matter disease (Manolio et al., 1999; Ryan et al., 2015; Saba et al., 2015; Saba et al., 2017), migraine with aura, and mesial temporal sclerosis (Cucchiara et al., 2013; Kuyumcu et al., 2017). Interestingly, the causative role of cerebrovascular variants in hypertension was assumed, warranting further research in this field (Warnert et al., 2016).

Although the importance of vascular variants has been discussed and widely researched, their developmental background remains unclear. Variations of the posterior communicating artery (PCoA) are heritable in contrast to other $\mathrm{CW}$ variants according to the results of a recent family study conducted on families with intracranial aneurysms (Sanchez van Kammen et al., 2018). Other results suggested the genetic background of CW variants ( $\mathrm{Du}$ et al., 2011; Faber et al., 2018; Li et al., 2015). A number of other stochastic and environmental factors, such as maternal preeclampsia, might alter the development of cerebrovascular variants (Luna et al., 2016), and pre-maturity might affect the formation of CW variants (Malamateniou et al., 2009). Thus, assessing the genetic versus environmental origin of CW variants could also provide knowledge of genetic predispositions for offspring and could help to understand the role of modifiable environmental factors. Accordingly, the aim of this twin study was to assess whether heritability plays a significant role in the formation of the variants of $\mathrm{CW}$ or they are influenced mostly by other (environmental or stochastic) factors.

\section{Methods}

Our study population consisted of healthy Caucasian twins who are members of the Hungarian Twin Registry (Littvay et al., 2013). The investigation was approved by the regional ethical committees (Regional/Local Committee of Sci- ence and Research Ethics Borsod-Abaúj-Zemplén, Heves and Nógrád County, approval number: 1046-260/2014, 2014/09/22 as well as Semmelweis University Regional and Institutional Committee of Science and Research Ethics, approval number: 189/2014, 2014/10/21). All participating twins signed an informed consent. The tenets of the Declaration of Helsinki were followed. A multiple self-reported questionnaire, based on a seven-part, self-reported response, was used to maximize the accuracy of zygosity classification (Heath et al., 2003). Detailed history and risk factors were obtained by a questionnaire, collecting data on body weight and height, body mass index (BMI), smoking, hypertension, and hypercholesterolemia. Both former and current smokers were included in the smoking group.

The twins underwent 3D Time-of-Flight magnetic resonance angiography (TOF MRA) of the cerebral arteries if the magnetic resonance imaging (MRI) study was not contraindicated. Patients were excluded with any of the following conditions: pregnancy or positive pregnancy test, breast-feeding, immunosuppressive or immunomodulant therapy including systemic steroids in the past 30 days, chemotherapy within 1 year, major surgery within 2 months, and transfusion or blood products received within 2 months. Moreover, pacemaker, implantable cardioverterdefibrillator or other implanted devices, magnetic metal foreign bodies, claustrophobia, and aphasia were regarded as exclusion criteria. The studies were performed in part at the Borsod County University Teaching Hospital, Miskolc, Hungary, and partly at the Semmelweis University Magnetic Resonance Research Centre. Members of a twin pair were examined on the same day by a professional operator.

T1-weighted coronal, T2-weighted sagittal, axial traceweighted diffusion, axial apparent diffusion coefficient, axial proton density, and axial T2 dark fluid images of the brain were obtained. TOF MR angiograms were obtained on the cranial vessels. No contrast agent was applied. The 3D multi-slab TOF MR angiograms were obtained and reconstructed with maximal intensity projection (MIP). At the first study location in the Borsod County University Teaching Hospital, measurements were performed using a Siemens Magnetom Verio 3 Tesla workstation (Siemens Healthcare $\mathrm{GmbH}$, Erlangen, Germany). The following imaging parameters were used: RT/ET $21 / 3.43 \mathrm{~ms}$, flip angle $18^{\circ}, 768 \times 768$ matrix, $0.5 \mathrm{~mm}$ slice thickness. At the second study location, a Philips Ingenia 1.5 Tesla workstation (Philips Healthcare, Best, the Netherlands) was used with the following imaging parameters: RT/ET 23/3.45 ms, flip angle $18^{\circ}, 552 \times 332$ matrix, $1.2 \mathrm{~mm}$ slice thickness. The twins also underwent transcranial Doppler sonography (TCD) study, which is an ultrasound-based method to assess real-time flow parameters in intracranial vessels. TCD was performed by the same investigator with the same ultrasound system (Multi-Dop T, Compumedics Germany $\mathrm{GmbH}$, Singen, Germany) with a $2 \mathrm{MHz}$ transducer. Through the temporal and transforaminal windows, 


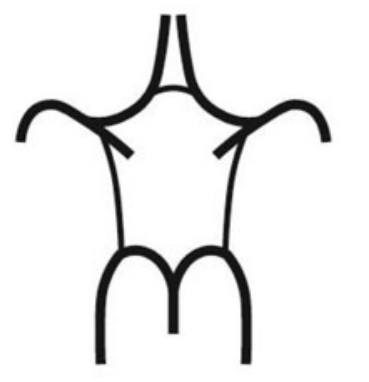

A

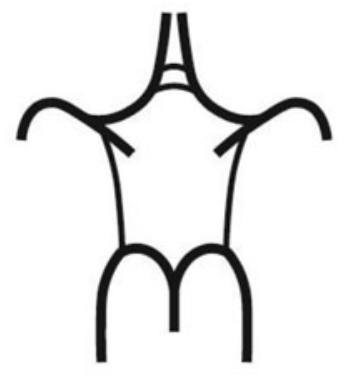

B
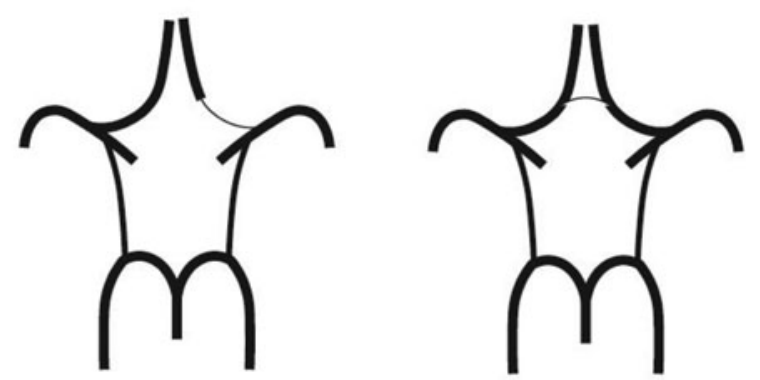

C

D

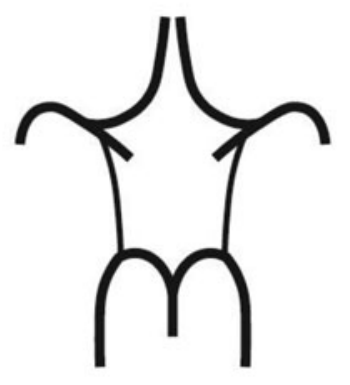

E

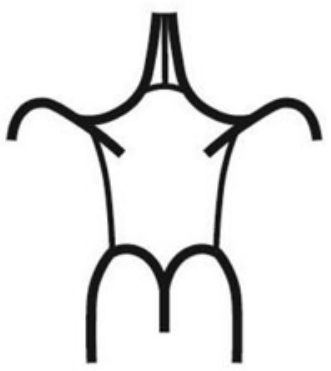

F

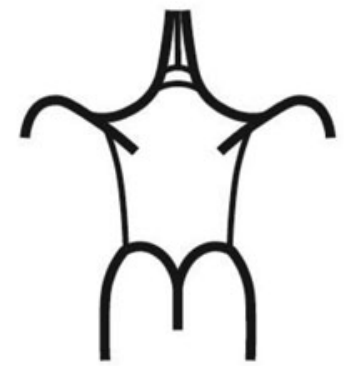

G

\section{FIGURE 1}

Variations of the anterior part of the circle of Willis. (A) "Normal" variant with single, non-hypoplastic ACoA and ACAs. (B) Two ACoAs. (C) Unilateral ACA A1 segment hypoplasia. (D) Hypoplastic ACoA. (E) Absent ACoA. (F) Medial artery of the corpus callosum arising from the ACoA. (G) Two ACoAs with medial artery of the corpus callosum arising from the ACoA. ACA = anterior cerebral artery, $A C \circ A=$ anterior communicating artery.

mean flow velocity (MFV) and pulsatility index (PI) were recorded bilaterally from the M1 segment of the middle cerebral artery (MCA), the A1 segment of the anterior cerebral artery (ACA), P1, P2a, and P2b segments of the posterior cerebral artery (PCA), and basilar artery. MFV is calculated as the end-diastolic velocity (EDV) plus one-third of the difference between peak systolic velocity (PSV) and EDV (Bathala et al., 2013). It is a useful marker of stenosis or occlusion of intracranial vessels. PI is used to assess cerebrovascular resistance and is calculated by subtracting EDV from PSV and dividing the value by MFV (Bathala et al., 2013). Flow direction was determined on all the aforementioned vascular segments, which was normal in all individuals.

Evaluation of the CW was made both on MIP and multislice MR images. A single observer performed all measurements in the project. This observer was blinded to zygosity and all clinical information. We investigated CW variants from two points of view (Hartkamp et al., 1999; Hoksbergen, Majoie et al., 2003; Li et al., 2011). First, we analyzed each vessel of the CW and determined whether they were non-hypoplastic, hypoplastic, or absent. Vessel diameters were measured on transverse slices of the 3D multi- slab TOF MR angiograms. We used a forced-choice method to define hypoplasia and absence of the vessels. Non-visible vessels were defined as absent (Hoksbergen, Majoie et al., 2003). Visible arteries with a diameter under $0.8 \mathrm{~mm}$ were defined as hypoplastic (Hartkamp et al., 1999). Second, we categorized the anterior and posterior $\mathrm{CW}$ variants separately according to their appearance. We used the morphological classification described by Hartkamp et al. (1999) and Li et al. (2011) as a basis. However, we made some modifications in their categories because some were not applicable to our findings. Figures 1 and 2 show our classification of the anterior and posterior $\mathrm{CW}$ variants.

Twin studies aim to determine the genetic and environmental basis of a certain phenotypic variance. The concept of twin studies is based on the assumption that monozygotic (MZ) twins share $100 \%$ of their genes on average, whereas dizygotic (DZ) twins share $50 \%$ of their genes similarly to non-twin siblings. The similarity between co-twins is estimated with the concordance rate in case of categorical variables (which were used in the current study for CW variants). If $\mathrm{MZ}$ twins have more similar phenotypes (and thus higher concordance rate) than DZ twins, then there is a bigger contribution to the phenotypic variance by genetic 


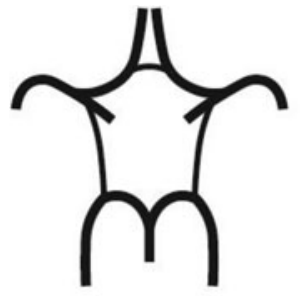

A
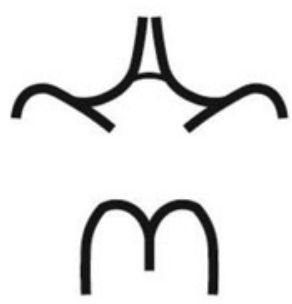

E

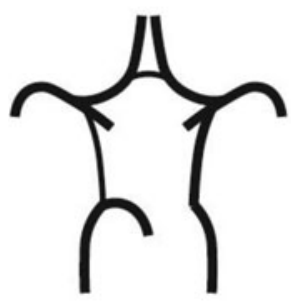

I

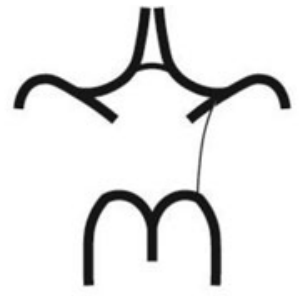

B

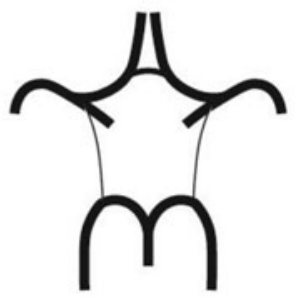

F

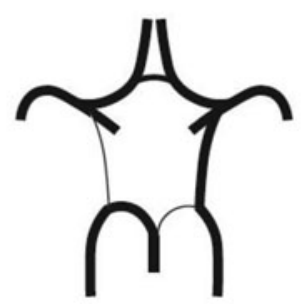

J

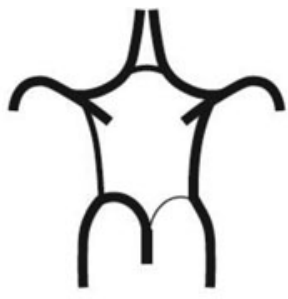

C

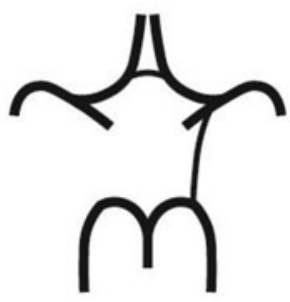

G

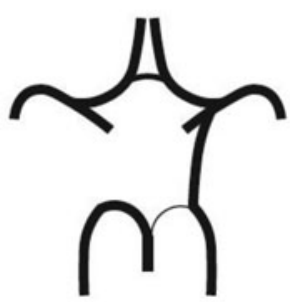

K

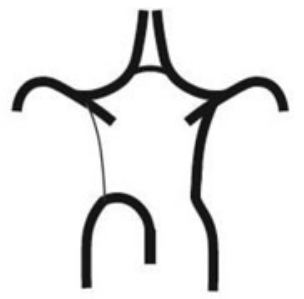

D

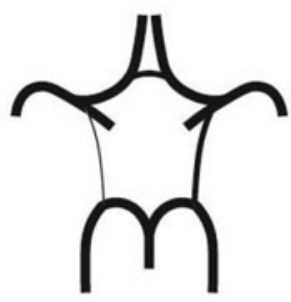

H

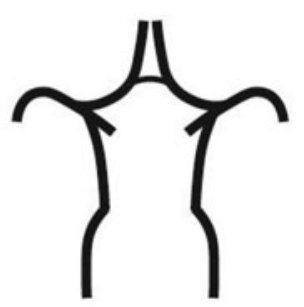

L

\section{FIGURE 2}

Variants of the posterior part of the circle of Willis. (A) "Normal" configuration with non-hypoplastic PCoAs present on both sides. (B) PCoA hypoplasia on one side, absent contralateral PCoA. (C) Unilateral fetal-type variant with ipsilateral dominant PCoA and hypoplastic PCA P1 segment. (D) Unilateral fetal-type variant with absent ipsilateral PCA P1 segment and contralateral PCoA hypoplasia. (E) Bilaterally absent PCoAs. (F) Bilaterally hypoplastic PCoAs. (G) Unilaterally absent PCoA. (H) Unilaterally hypoplastic PCoA. (I) Unilateral fetal-type variant with ipsilateral absent PCA P1 segment. (J) Unilateral fetal-type variant with ipsilateral hypoplastic PCA P1 segment and contralateral hypoplastic PCoA. (K) Unilateral fetal-type variant with ipsilateral hypoplastic PCA P1 segment and contralateral absent PCoA. (L) Bilateral fetal-type variant with bilaterally absent PCA P1 segments. PCA $=$ posterior cerebral artery, $P C \circ A=$ posterior communicating artery.

effects. Furthermore, twins share the same early environment, and therefore, common and non-common environmental factors can be estimated. In the current study, this detailed analysis was not possible due to the small study population. Therefore, concordance and discordance rates were calculated (Sahu \& Prasuna, 2016).

We determined the concordance rates of anterior and posterior CW morphologies separately. Furthermore, we calculated concordance rates for the presence of hypoplastic or absent vessels in the CW. Pairwise concordance rates were estimated by the following formula (Witte et al., 1999):

$$
\frac{n \text { concordant twin pairs }}{n \text { concordant twin pairs }+n \text { discordant twin pairs }}
$$

A pair of twins was defined as concordant if both members of the pair had any kind of variant CW anatomy. In discordant twin pairs, one member of the twin pair had classical CW anatomy and the other member had variant anatomy in the CW. Both anterior and posterior CW parts were investigated separately according to these categories.

Comparison of the $\mathrm{MZ}$ and $\mathrm{DZ}$ groups regarding BMI and age was evaluated with an independent samples $t$ test. Similarly, an independent samples $t$ test was used to compare the BMI among MZ discordant twins with and without $\mathrm{CW}$ variants. A chi-square test was applied to investigate differences between $M Z$ and $D Z$ twins and $M Z$ discordant twins for smoking, diabetes, hypertension, and hypercholesterolemia. We analyzed intrapair differences in the MZ discordant group regarding flow parameters recorded with TCD using paired $t$ tests. Statistical Package for the Social Sciences (SPSS) software (IBM SPSS Statistics for Windows, Version 24.0, Released 2016, IBM Corp., Armonk, New York) was used for statistical analysis.

\section{Results}

A total of 122 twins attended the study (61 pairs), of whom 39 pairs were $M Z$ and 22 were $\mathrm{DZ}$ after the exclusion of 4 
TABLE 1

Baseline Characteristics of Our Study Population

\begin{tabular}{lllll}
\hline & Total $(N=122)$ & $\mathrm{MZ}(n=78)$ & $\mathrm{DZ}(n=44)$ & $p$ value \\
\hline Zygosity (MZ:DZ) & $78: 44$ & - & - & - \\
Sex (male:female) & $40: 82$ & $28: 50$ & $12: 32$ & .33 \\
Age (years) & $49.7 \pm 13.4$ & $48.4 \pm 14.4$ & $52.0 \pm 11.0$ & .16 \\
BMI & $26.8 \pm 8.1$ & $26.4 \pm 9.4$ & $40.4 \pm 27.4$ & .56 \\
Smoking, $n(\%)$ & $24(23.1)$ & $16(24.2)$ & $8(21.1)$ & .71 \\
Diabetes, $n(\%)$ & $10(9.6)$ & $4(6.1)$ & $6(15.8)$ & .11 \\
Hypertension, $n(\%)$ & $28(26.9)$ & $15(22.7)$ & $13(34.2)$ & .20 \\
Hypercholesterolemia, $n(\%)$ & $16(16.2)$ & $6(9.2)$ & $10(29.4)$ & .01
\end{tabular}

Note: $\mathrm{BMI}=$ body mass index; $\mathrm{DZ}=$ dizygotic; $\mathrm{MZ}=$ monozygotic.

Data are shown as mean \pm standard deviation with continuous variables.

\section{TABLE 2}

Concordance Rates of the Anterior and Posterior Anatomies of the Circle of Willis According to Morphological Aspects

\begin{tabular}{|c|c|c|c|c|c|c|c|}
\hline & & & & \multicolumn{2}{|c|}{ Twin A } & \multirow{2}{*}{$\begin{array}{l}\text { Number of } \\
\text { concordant/discordant } \\
\text { pairs }\end{array}$} & \multirow{2}{*}{$\begin{array}{l}\text { Pairwise } \\
\text { concordance rate }\end{array}$} \\
\hline & & & & Normal CW & Variant CW & & \\
\hline \multirow[t]{4}{*}{ Anterior CW } & Twin B & $M Z$ & Normal CW & 12 & 13 & $6 / 21$ & 0.22 \\
\hline & & & Variant CW & 8 & 6 & & \\
\hline & & $\mathrm{DZ}$ & Normal CW & 7 & 3 & $7 / 8$ & 0.47 \\
\hline & & & Variant CW & 5 & 7 & & \\
\hline \multirow[t]{4}{*}{ Posterior CW } & Twin B & $M Z$ & Normal CW & 3 & 4 & $25 / 11$ & 0.69 \\
\hline & & & Variant CW & 7 & 25 & & \\
\hline & & $\mathrm{DZ}$ & Normal CW & 2 & 1 & $17 / 3$ & 0.85 \\
\hline & & & Variant CW & 2 & 17 & & \\
\hline
\end{tabular}

Note: $C W=$ circle of Willis; $\mathrm{DZ}=$ dizygotic; $M Z$ = monozygotic.

twin pairs. These twin pairs had to be excluded either because they did not show up at the scheduled appointment for MRI (three pairs) or because of claustrophobia (one pair). The average age was 48.4 and 52.0 years in the $M Z$ and DZ groups, respectively. There was no significant difference regarding average age between the two groups $(p=.16)$. Similarly, no significant difference was observed between the $\mathrm{MZ}$ and $\mathrm{DZ}$ groups regarding anthropometric variables and risk factors, except for hypercholesterolemia $(p=0.01)$. Table 1 shows the baseline characteristics of our population. Supplementary Tables S1 and S2 show the prevalence of variants of the anterior and posterior CW. The most common anterior $\mathrm{CW}$ configuration was the "normal" form of the $\mathrm{CW}$ without hypoplasticity or absence in the ACA and anterior communicating artery (ACoA) (A, 45.0\%) followed by hypoplastic ACoA (D, 25.8\%) and absent ACoA (E, 10.8\%). Among posterior CW configurations, bilaterally missing PCoA (E, 22.5\%) was the most commonly observed variant. Normal morphology (A, 17.5\%), bilaterally hypoplastic PCoA (F, 15.0\%), and unilaterally hypoplastic PCoA $(\mathrm{H}, 11.7 \%)$ were also among the most common configurations. The total percentage of unilateral fetal configuration (C, D, I, J, and K) was seen in $10.9 \%$. No significant difference was observed regarding anterior and posterior CW variants shown in Figures 1 and 2 between the MZ and DZ groups with one exception: the bilaterally hypoplastic PCoA (F variant) appeared in a significantly higher rate in the DZ group $(p=.02)$.
Table 2 shows the concordance and discordance rates of the anterior and posterior $\mathrm{CW}$ variants according to the morphological classification. MZ concordance was not higher than DZ concordance in anterior and posterior variants, which presumes the dominance of environmental factors on CW configurations. Figures 3 and 4 show examples of differences in CW configurations in MZ twins.

Out of our MZ twin group, 23 twin pairs were discordant regarding anterior or posterior $\mathrm{CW}$ variants. We analyzed which environmental effect could have a significant impact on the development of anterior or posterior $\mathrm{CW}$ variants in discordant MZ twin pairs. Smoking, diabetes, hypertension, and hypercholesterolemia did not differ significantly between MZ discordant twins with variant and classical CW configuration (Supplementary Table S3). Flow parameters, such as MFV and PI, did not show a significant difference within twin pairs of the MZ discordant group in any segment of the CW (Supplementary Table S4) in our healthy subjects, except for the right ACA.

\section{Discussion}

Our results suggest that $\mathrm{CW}$ variants seem to be determined by environmental factors rather than genetic effects. The presence of anterior and posterior CW variants was not significantly associated with current BMI, hypertension, and hypercholesterolemia, suggesting that these factors do not affect the development of the cerebrovascular 

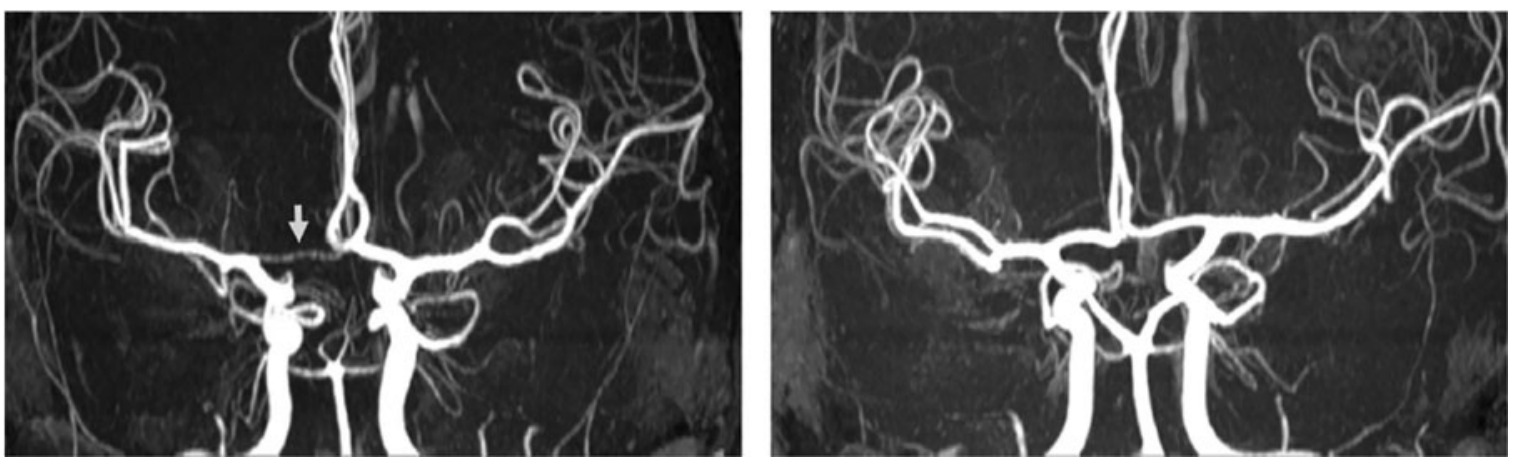

\section{FIGURE 3}

Differences in anterior CW variants of a monozygotic twin pair. Twin A has a right-sided hypoplastic ACA A1 segment (arrow). Twin B has normal anterior $\mathrm{CW}$ configuration. $\mathrm{ACA}=$ anterior cerebral artery, $\mathrm{CW}=$ circle of Willis.
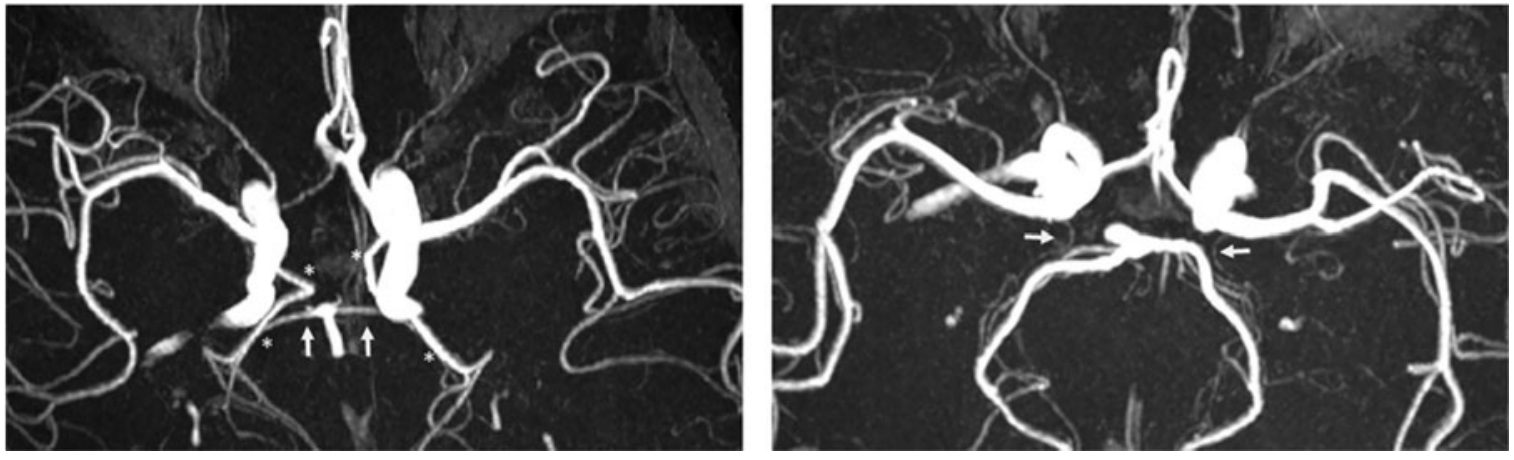

\section{FIGURE 4}

Differences in posterior CW variants of the same monozygotic twin pair as shown in Figure 3. Twin A has bilateral fetal configuration (asterisk) with absent PCA P1 segments on both sides. The arrows in the left picture point to the superior cerebellar arteries. Twin B has bilaterally absent PCoAs. The arrows in the right picture show the anterior choroidal arteries. PCA $=$ posterior cerebral artery, $\mathrm{PC} \circ \mathrm{A}=$ posterior communicating artery, $\mathrm{CW}=$ circle of Willis.

variants. Also, there was no significant difference regarding cerebral blood flow in MZ twins discordant to $\mathrm{CW}$ variants, indicating that these variants may not alter the flow in healthy individuals.

In a recent study, heritability of $\mathrm{CW}$ variants was assessed for the first time in humans in the frame of a family study (Sanchez van Kammen et al., 2018). According to the results, the occurrence of PCoA was significantly higher within families with intracerebral aneurysms, and this was not the case regarding ACA A1 asymmetry, fetal configuration, and the classical morphology (Sanchez van Kammen et al., 2018). Comparing these findings to our results is challenging due to the different study designs and because our study included healthy individuals, but it is unlikely that genetic pre-disposition is present for only one variant. Furthermore, it is also possible that the findings are simply due to the higher general prevalence of PCoA variants, since hypoplasia or absence of PCoA is the most commonly observed CW variant (De Silva et al., 2011; Eftekhar et al., 2006; Hashemi et al., 2013; Klimek-Piotrowska et al., 2013).
What environmental factors may be behind CW variants is yet to be examined. Our results show that environmental factors may determine CW anatomy; however, we could not confirm any significant association between classical cardiovascular risk factors at study time and the presence of $\mathrm{CW}$ variants. Our volunteer twin population consisted of healthy individuals. Results on the association of cardiovascular risk factors and $\mathrm{CW}$ variants are controversial in the literature. A recent animal study showed that chronically elevated blood pressure and aging decreased PCoA diameter in inbred mice, but obesity, hyperlipidemia, metabolic syndrome, and diabetes mellitus did not affect this arterial diameter (Faber et al., 2018). Jin et al. (2016) found that incomplete posterior circulation was present in subjects without hypertension more commonly than in those with hypertension. Although we did not investigate gender differences in CW variants, Zaninovich et al. (2017) found that complete CW differed significantly between men and women above the age of 39 years, suggesting that age combined with other environmental factors may have a significant impact. Another study did not find any significant 
association between cardiovascular risk factors and the number of hypoplastic arteries in the CW, in line with our findings, except for increasing age (Gutierrez et al., 2013). Significant differences among age groups were described, with incomplete CW being more common with increasing age (El-Barhoun et al., 2009; Li et al., 2011; Zaninovich et al., 2017). Our population was relatively young (average age $50.4 \pm 13.6$ years), and therefore, the prevalence of CW variants may not be high enough for comparison. Our findings could also be explained by the small population size, which limited us to perform a more detailed heritability analysis.

The high number of MZ discordant twins may be explained by epigenetic effects. These interactions between the genes and the environment might lead to changes in phenotypes. Although twins share the same uterus, it does not necessarily mean that they share the same in-utero environment, since the fetoplacental unit might be unique in each member of the twin pair (Czyz et al., 2012). Hypoxia and metabolic effects could have an influence on CW variants already at intrauterine development and in adult life. Changes in the volume of the amniotic fluid, blood flow, and hemodynamic circumstances during brain development were shown to influence calibers of the intracranial vessels (Hillen, 1986; Mari et al., 1992; Van Overbeeke et al., 1991). The findings of Hartkamp et al. (1999) underline the importance of hemodynamic and metabolic factors later in adult life as patients with ICA stenosis of 7099\% or occlusion of the ICA tend to have complete CW on the side of the stenosis or occlusion, and this corresponds with the results of a study conducted on patients who underwent carotid endarterectomy or stenting, which demonstrated that 1 month after the operation (once the need for collateral supply decreased), collaterals of the CW became reduced in size (Bost et al., 2014).

Term and pre-term birth (Malamateniou et al., 2009), intrauterine circumstances, and certain diseases during pregnancy may also influence CW configurations of the fetus. Pre-eclampsia leads to deficiency in placental growth factor (PGF), which has an impact on cerebrovascular development in mice: Unilaterally hypoplastic CW and a decreased number of collaterals were noted in PGF-/- mice (Luna et al., 2016). These results should stimulate further research regarding the presence of $\mathrm{CW}$ variations early in life. Future studies should investigate whether circumstances of delivery (e.g., cardiotocography monitoring or data regarding cesarean section) are associated with the presence of CW variants.

Li et al. (2015) identified four genes in gerbils that could possibly play a role in the formation of CW variants. Another study, also conducted on gerbils, investigated CW variants through generations and concluded that configurations of ACoA are heritable, mainly from the mother's side, which was not shown in the case of posterior variants (Du et al., 2011). On the other hand, Beckmann (2000) described individual differences among mice within the same strain. The differences mainly involve the presence of PCoA (Beckmann, 2000), which corresponds with our findings in humans. Since our findings do not support genetic pre-disposition to $\mathrm{CW}$ variants, we speculate whether epigenetic effects could have a more significant influence. Epigenetic effects play a substantial role in the evolution of different phenotypes, as described by the Waddington (1957) epigenetic landscape metaphor. According to this theory, the development of cells can be regarded as a pathway with several junctions leading to different phenotypes. It is possible that the various CW variants are the results, at least partly, of a similar process. There are genetic processes that initiate development of cerebral arteries, but as the occipital lobe grows in the human brain, its functional demand leads to hemodynamic changes, and thus, CW variations may arise as consequence of these effects (Van Overbeeke et al., 1991). This is in line with the concept of angioadaptation, which is an adaptive change of the vessel wall structure and its continuous interaction with the environment due to hemodynamic, metabolic, and molecular inputs (le Noble et al., 2008; Zakrzewicz et al., 2002). Thus, the presence of variants could depend heavily on the development of the arteries (Lasjaunias et al., 2001). Furthermore, there might be developmental interactions between the CW and the skull structure, as described by Jamniczky and Hallgrimsson (2011). The findings of Faber et al. (2018) in mice underline the importance of random stochastic factors on PCoA variants in the developmental phase, since low birth weight was not significantly associated with the presence of CW variants. Aging and hypertension, but not other cardiovascular risk factors, influenced PCoA diameter significantly, and the PCoA configuration differed among mice strains with different genetic background (Faber et al., 2018). Thus, it is suggested in this article that genetic and stochastic factors are the primary determinants of PCoA configuration, which is then further affected by environmental factors later in life (Faber et al., 2018).

According to our results, the presence of CW variants does not influence the cerebral flow among healthy individuals with non-pathologic circumstances, underlying the fact that the clinical manifestation of the presence of $\mathrm{CW}$ variants is expected in low cerebral blood flow states, such as in ICA stenosis or occlusion (Hoksbergen, Legemate et al., 2003; Park et al., 2018). Recent results pointed out the association between compromized CW and brain ischemia in a population with high-grade ICA stenosis (Varga et al., 2018), and especially the failure of collateral flow may predispose to ischemic events (Hoksbergen et al., 2003; Varga et al., 2018). TCD and MRA findings have not been compared in healthy individuals until this report; however, findings of these two modalities correlated in patients with intracerebral arterial pathology (Han et al., 2014; Hendrikse et al., 2008). 
Our study has limitations that have to be acknowledged. The relatively small population may influence our results, which limited us to perform complex genetic analysis, identifying the common and unique environmental factors due to low power (ACE modeling). The comparison of concordance rates alone does not allow conclusions regarding heritability. A high concordance does not mean that the phenotype is heritable, and conversely, low concordance rates do not exclude genetic effects (van Dongen et al., 2012). Our imaging method (TOF MRA) can be regarded as a limitation factor of this study because the longer acquisition time increases the motion artefacts on a TOF MRA image. Contrast-enhanced MRI has better resolution and is more sensitive to detect the CW variants, but it is an invasive procedure and requires contrast administration, which is ethically not acceptable in a healthy volunteer population. Moreover, previous studies found no significant difference between contrast-enhanced MRA and TOF MRA regarding displaying intracranial arteries (Alfke et al., 2011; Heiserman et al., 1992). Detecting ACA, MCA, and PCA with TOF MRA has a sensitivity of $94-100 \%$ compared with the gold standard method, i.e., digital subtraction angiography. The value is lower (65\%) in case of anterior and posterior communicating arteries (Stock et al., 1996). This may explain why in our study population absent or missing ACoA and PCoA were the most common variants. TOF MRA is useful in the diagnosis of stenoocclusive disease as sensitivity and specificity of TOF MRA of the MCA compared to conventional angiography were $88 \%$ and $97 \%$, respectively (Korogi et al., 1994). In addition, contrast-enhanced MRA has the risk of gadolinium accumulation in the brain and bone tissue, even in patients with normal renal function (Kanda et al., 2016), and therefore, it was not an option in the current study.

\section{Conclusion}

Our study is the first to investigate genetic versus environmental background of the CW variants with MRI in the frame of a twin study. Our results show that anatomical variants of the $\mathrm{CW}$, which commonly occur and might predispose to cerebrovascular events, might be shaped and influenced by environmental or stochastic factors; however, our results do not allow us to draw conclusions on heritability. Anterior and posterior $\mathrm{CW}$ variants were not associated with current BMI, smoking, hypertension and hypercholesterolemia, and altered blood flow using TCD. Since CW variants are known risk factors of cerebrovascular ischemia, determining their origin is essential to develop preventive strategies. Our results should stimulate further research on the origin of $\mathrm{CW}$ variants in larger twin populations.

\section{Acknowledgments}

No financial support can be stated. The authors acknowledge the help of MR operators. The au- thors thank Dr Philip Auyang for English language editing.

\section{Disclosure of Interest}

None.

\section{Supplementary material}

To view supplementary material for this article, please visit https://doi.org/10.1017/thg.2018.50

\section{References}

Alfke, K., Jensen, U., Pool, C., Rohr, A., Bruning, R., Weber, J., ... Jansen, O. (2011). Contrast-enhanced magnetic resonance angiography in stroke diagnostics: Additional information compared with time-of-flight magnetic resonance angiography? Clinical Neuroradiology, 21, 5-10.

Bathala, L., Mehndiratta, M. M., \& Sharma, V. K. (2013). Transcranial doppler: Technique and common findings (Part 1). Annals of Indian Academy of Neurology, 16, 174-179.

Beckmann, N. (2000). High resolution magnetic resonance angiography non-invasively reveals mouse strain differences in the cerebrovascular anatomy in vivo. Magnetic Resonance in Medicine, 44, 252-258.

Bisschops, R. H., Klijn, C. J., Kappelle, L. J., van Huffelen, A. C., \& van der Grond, J. (2003). Collateral flow and ischemic brain lesions in patients with unilateral carotid artery occlusion. Neurology, 60, 1435-1441.

Bost, R. B., Hendrikse, J., Algra, A., de Borst, G. J., Kappelle, L. J., Jongen, L. M., ... van der Worp, H. B. (2014). Effects of carotid endarterectomy or stenting on arterial diameters in the circle of Willis. Journal of Stroke \& Cerebrovascular Diseases, 23, 699-705.

Cucchiara, B., Wolf, R. L., Nagae, L., Zhang, Q., Kasner, S., Datta, R., ... Detre, J. A. (2013). Migraine with aura is associated with an incomplete circle of Willis: Results of a prospective observational study. PLoS One, 8, e71007.

Czyz, W., Morahan, J. M., Ebers, G. C., \& Ramagopalan, S. V. (2012). Genetic, environmental and stochastic factors in monozygotic twin discordance with a focus on epigenetic differences. BMC Medicine, 10, 93.

De Silva, K. R., Silva, R., Amaratunga, D., Gunasekera, W. S., \& Jayesekera, R. W. (2011). Types of the cerebral arterial circle (circle of Willis) in a Sri Lankan population. BMC Neurology, 11, 5.

Du, X. Y., Zhu, X. D., Dong, G., Lu, J., Wang, Y., Zeng, L., ... Chen, Z. W. (2011). Characteristics of circle of Willis variations in the Mongolian gerbil and a newly established ischemia-prone gerbil group. ILAR Journal, 52, E1-E7.

Eftekhar, B., Dadmehr, M., Ansari, S., Ghodsi, M., Nazparvar, B., \& Ketabchi, E. (2006). Are the distributions of variations of circle of Willis different in different populations? - Results of an anatomical study and review of literature. BMC Neurology, 6, 22.

El-Barhoun, E. N., Gledhill, S. R., \& Pitman, A. G. (2009). Circle of Willis artery diameters on MR angiography: An 
Australian reference database. Journal of Medical Imaging and Radiation Oncology, 53, 248-260.

Faber, J. E., Zhang, H., Rzechorzek, W., Dai, K. Z., Summers, B. T., Blazek, C., \& Hedges, S. J. (2018). Genetic and environmental contributions to variation in the posterior communicating collaterals of the circle of Willis. Translational Stroke Research. Advance online publication.

Gutierrez, J., Sultan, S., Bagci, A., Rundek, T., Alperin, N., Elkind, M. S., ... Wright, C. B. (2013). Circle of Willis configuration as a determinant of intracranial dolichoectasia. Cerebrovascular Diseases, 36, 446-453.

Han, C., Feng, H., Han, Y. Q., Liu, W. W., Zhang, Z. S., Yang, W. Z., \& Duan, L. (2014). Prospective screening of family members with moyamoya disease patients. PLoS One, 9, e88765.

Hartkamp, M. J., van Der Grond, J., van Everdingen, K. J., Hillen, B., \& Mali, W. P. (1999). Circle of Willis collateral flow investigated by magnetic resonance angiography. Stroke, 30, 2671-2678.

Hashemi, S. M., Mahmoodi, R., \& Amirjamshidi, A. (2013). Variations in the anatomy of the Willis circle: A 3-year cross-sectional study from Iran (2006-2009). Are the distributions of variations of circle of Willis different in different populations? Result of an anatomical study and review of literature. Surgical Neurology International, 4, 65.

Heath, A. C., Nyholt, D. R., Neuman, R., Madden, P. A., Bucholz, K. K., Todd, R. D., ... Martin, N. G. (2003). Zygosity diagnosis in the absence of genotypic data: An approach using latent class analysis. Twin Research, 6, 22-26.

Heiserman, J. E., Drayer, B. P., Keller, P. J., \& Fram, E. K. (1992). Intracranial vascular stenosis and occlusion: Evaluation with three-dimensional time-of-flight MR angiography. Radiology, 185, 667-673.

Henderson, R. D., Eliasziw, M., Fox, A. J., Rothwell, P. M., \& Barnett, H. J. (2000). Angiographically defined collateral circulation and risk of stroke in patients with severe carotid artery stenosis. North American Symptomatic Carotid Endarterectomy Trial (NASCET) Group. Stroke, 31, 128-132.

Hendrikse, J., Hartkamp, M. J., Hillen, B., Mali, W. P., \& van der Grond, J. (2001). Collateral ability of the circle of Willis in patients with unilateral internal carotid artery occlusion: Border zone infarcts and clinical symptoms. Stroke, $32,2768-2773$.

Hendrikse, J., Klijn, C. J., van Huffelen, A. C., Kappelle, L. J., \& van der Grond, J. (2008). Diagnosing cerebral collateral flow patterns: Accuracy of non-invasive testing. Cerebrovascular Diseases, 25, 430-437.

Hillen, B. (1986). The variability of the circle of Willis: Univariate and bivariate analysis. Acta morphologica NeerlandoScandinavica, 24, 87-101.

Hoksbergen, A. W., Legemate, D. A., Csiba, L., Csati, G., Siro, P., \& Fulesdi, B. (2003). Absent collateral function of the circle of Willis as risk factor for ischemic stroke. Cerebrovascular Diseases, 16, 191-198.

Hoksbergen, A. W., Majoie, C. B., Hulsmans, F. J., \& Legemate, D. A. (2003). Assessment of the collateral function of the circle of Willis: Three-dimensional time-of-flight MR angiography compared with transcranial color-coded duplex sonography. American Journal of Neuroradiology, 24, 456462.

Jamniczky, H. A., \& Hallgrimsson, B. (2011). Modularity in the skull and cranial vasculature of laboratory mice: Implications for the evolution of complex phenotypes. Evolution \& Development, 13, 28-37.

Jin, Z. N., Dong, W. T., Cai, X. W., Zhang, Z., Zhang, L. T., Gao, F., ... Yang, X. Y. (2016). CTA characteristics of the circle of Willis and intracranial aneurysm in a Chinese crowd with family history of stroke. BioMed Research International, 2016, Article ID 1743794.

Kanda, T., Oba, H., Toyoda, K., Kitajima, K., \& Furui, S. (2016). Brain gadolinium deposition after administration of gadolinium-based contrast agents. Japanese Journal of Radiology, 34, 3-9.

Klimek-Piotrowska, W., Kopec, M., Kochana, M., Krzyzewski, R. M., Tomaszewski, K. A., Brzegowy, P., \& Walocha, J. (2013). Configurations of the circle of Willis: A computed tomography angiography based study on a Polish population. Folia Morphologica (Warsz), 72, 293-299.

Kluytmans, M., van der Grond, J., van Everdingen, K. J., Klijn, C. J., Kappelle, L. J., \& Viergever, M. A. (1999). Cerebral hemodynamics in relation to patterns of collateral flow. Stroke, 30, 1432-1439.

Korogi, Y., Takahashi, M., Mabuchi, N., Miki, H., Shiga, H., Watabe, T., ... Fujiwara, S. (1994). Intracranial vascular stenosis and occlusion: Diagnostic accuracy of threedimensional, Fourier transform, time-of-flight MR angiography. Radiology, 193, 187-193.

Kuyumcu, G., Byrne, R. W., Dawe, R. J., \& Kocak, M. (2017). Incomplete circle of Willis: A risk factor for mesial temporal sclerosis? Epilepsy Research, 132, 29-33.

Lasjaunias, P., Berenstein, A., \& ter Brugge, K. G. (Eds.) (2001). Surgical neuroangiography, Vol. 1. Clinical vascular anatomy and variations (2nd ed.). Berlin Heidelberg: Springer-Verlag.

le Noble, F., Klein, C., Tintu, A., Pries, A., \& Buschmann, I. (2008). Neural guidance molecules, tip cells, and mechanical factors in vascular development. Cardiovascular Research, 78, 232-241.

Li, Q., Li, J., Lv, F., Li, K., Luo, T., \& Xie, P. (2011). A multidetector CT angiography study of variations in the circle of Willis in a Chinese population. Journal of Clinical Neuroscience, 18, 379-383.

Li, Z., Huo, X., Zhang, S., Lu, J., Li, C., Guo, M., ... Chen, Z. (2015). Selection of genes associated with variations in the circle of Willis in gerbils using suppression subtractive hybridization. PLoS One, 10, e0127355.

Littvay, L., Metneki, J., Tarnoki, A. D., \& Tarnoki, D. L. (2013). The Hungarian Twin Registry. Twin Research and Human Genetics, 16, 185-189.

Luna, R. L., Kay, V. R., Ratsep, M. T., Khalaj, K., Bidarimath, M., Peterson, N., ... Croy, B. A. (2016). Placental growth factor deficiency is associated with impaired cerebral vascular development in mice. Molecular Human Reproduction, 22, 130-142. doi: 10.1093/molehr/gav069 
Malamateniou, C., Adams, M. E., Srinivasan, L., Allsop, J. M., Counsell, S. J., Cowan, F. M., ... Rutherford, M. A. (2009). The anatomic variations of the circle of Willis in pretermat-term and term-born infants: An MR angiography study at 3T. American Journal of Neuroradiology, 30, 1955-1962.

Manolio, T. A., Burke, G. L., O’Leary, D. H., Evans, G., Beauchamp, N., Knepper, L., \& Ward, B. (1999). Relationships of cerebral MRI findings to ultrasonographic carotid atherosclerosis in older adults: The cardiovascular health study. CHS Collaborative Research Group. Arteriosclerosis, Thrombosis, and Vascular Biology, 19, 356-365.

Mari, G., Wasserstrum, N., \& Kirshon, B. (1992). Reduction in the middle cerebral artery pulsatility index after decompression of polyhydramnios in twin gestation. American Journal of Perinatology, 9, 381-384.

Morgenstern, L. B., Fox, A. J., Sharpe, B. L., Eliasziw, M., Barnett, H. J., \& Grotta, J. C. (1997). The risks and benefits of carotid endarterectomy in patients with near occlusion of the carotid artery. North American Symptomatic Carotid Endarterectomy Trial (NASCET) Group. Neurology, 48, 911-915.

Park, B. J., Kim, K. M., Lee, W. J., Chun, I. K., Kim, I., Lee, S. J., \& Kim, S. (2018). Clinical significance of the circle of Willis in patients with symptomatic internal carotid artery occlusion. World Neurosurgery, 115, e585-e591.

Qiu, C., Zhang, Y., Xue, C., Jiang, S., \& Zhang, W. (2015). MRA study on variation of the circle of Willis in healthy Chinese male adults. BioMed Research International, 2015, Article ID 976340.

Rutgers, D. R., Klijn, C. J., Kappelle, L. J., \& van der Grond, J. (2004). Recurrent stroke in patients with symptomatic carotid artery occlusion is associated with high-volume flow to the brain and increased collateral circulation. Stroke, $35,1345-1349$.

Ryan, D. J., Byrne, S., Dunne, R., Harmon, M., \& Harbison, J. (2015). White matter disease and an incomplete circle of Willis. International Journal of Stroke, 10, 547-552.

Saba, L., Raz, E., Fatterpekar, G., Montisci, R., di Martino, M., Bassareo, P. P., \& Piga, M. (2015). Correlation between leukoaraiosis volume and circle of Willis variants. Journal of Neuroimaging, 25, 226-231.

Saba, L., Sanfilippo, R., Porcu, M., Lucatelli, P., Montisci, R., Zaccagna, F., ... Wintermark, M. (2017). Relationship between white matter hyperintensities volume and the circle of Willis configurations in patients with carotid artery pathology. European Journal of Radiology, 89, 111-116.

Sahu, M., \& Prasuna, J. G. (2016). Twin studies: A unique epidemiological tool. Indian Journal of Community Medicine, 41, 177-182.

Sanchez van Kammen, M., Moomaw, C. J., van der Schaaf, I. C., Brown, R. D. Jr., Woo, D., Broderick, J. P., ... Ruigrok, Y. M. (2018). Heritability of circle of Willis variations in families with intracranial aneurysms. PLoS One, 13, e0191974.
Schomer, D. F., Marks, M. P., Steinberg, G. K., Johnstone, I. M., Boothroyd, D. B., Ross, M. R., ... Enzmann, D. R. (1994). The anatomy of the posterior communicating artery as a risk factor for ischemic cerebral infarction. New England Journal of Medicine, 330, 1565-1570.

Songsaeng, D., Geibprasert, S., Willinsky, R., Tymianski, M., TerBrugge, K. G., \& Krings, T. (2010). Impact of anatomical variations of the circle of Willis on the incidence of aneurysms and their recurrence rate following endovascular treatment. Clinical Radiology, 65, 895-901.

Stock, K. W., Wetzel, S., Kirsch, E., Bongartz, G., Steinbrich, W., \& Radue, E. W. (1996). Anatomic evaluation of the circle of Willis: MR angiography versus intraarterial digital subtraction angiography. American Journal of Neuroradiology, 17, 1495-1499.

Tegos, T. J., Kalodiki, E., Daskalopoulou, S. S., \& Nicolaides, A. N. (2000). Stroke: Epidemiology, clinical picture, and risk factors-Part I of III. Angiology, 51, 793-808.

van Dongen, J., Slagboom, P. E., Draisma, H. H., Martin, N. G., \& Boomsma, D. I. (2012). The continuing value of twin studies in the omics era. Nature Reviews Genetics, 13, 640653.

Van Overbeeke, J. J., Hillen, B., \& Tulleken, C. A. (1991). A comparative study of the circle of Willis in fetal and adult life. The configuration of the posterior bifurcation of the posterior communicating artery. Journal of Anatomy, 176, 45-54.

van Seeters, T., Hendrikse, J., Biessels, G. J., Velthuis, B. K., Mali, W. P., Kappelle, L. J., ... Group, S. S. (2015). Completeness of the circle of Willis and risk of ischemic stroke in patients without cerebrovascular disease. Neuroradiology, 57, 1247-1251.

Varga, A., Di Leo, G., Banga, P. V., Csobay-Novak, C., Kolossvary, M., Maurovich-Horvat, P., \& Huttl, K. (2018). Multidetector CT angiography of the circle of Willis: Association of its variants with carotid artery disease and brain ischemia. European Radiology. Advance online publication.

Waddington, C. H. (1957). The strategy of the genes (1st ed.). London: George Allen \& Unwin.

Warnert, E. A. H., Rodrigues, J. C. L., Burchell, A. E., Neumann, S., Ratcliffe, L. E. K., Manghat, N. E., ... Hart, E. C. (2016). Is high blood pressure self-protection for the brain? Circulation Research, 119, e140-e150.

Witte, J. S., Carlin, J. B., \& Hopper, J. L. (1999). Likelihoodbased approach to estimating twin concordance for dichotomous traits. Genetic Epidemiology, 16, 290-304.

Zakrzewicz, A., Secomb, T. W., \& Pries, A. R. (2002). Angioadaptation: Keeping the vascular system in shape. News in Physiological Sciences, 17, 197-201.

Zaninovich, O. A., Ramey, W. L., Walter, C. M., \& Dumont, T. M. (2017). Completion of the circle of Willis varies by gender, age, and indication for computed tomography angiography. World Neurosurgery, 106, 953-963. 